\title{
Investigation on nonlinear-optical properties of palm oil/silver nanoparticles
}

\section{R. Zamiri \\ R. Parvizi}

A. Zakaria

azmizak@gmail.com

\section{A. R. Sadrolhosseini \\ G. Zamiri}

M. Darroudi

\section{S. Husin}

Department of Physics, Faculty of Science, Universiti Putra Malaysia, 43400 UPM Serdang, Selangor, Malaysia

Photonics Laboratory, Department of Physics, University of Malaya 50603 Kuala Lumpur, Malaysia

Department of Physics, Faculty of Science, Universiti Putra Malaysia, 43400 UPM Serdang, Selangor, Malaysia

Department of Physics, Faculty of Science, Universiti Putra Malaysia, 43400 UPM Serdang, Selangor, Malaysia

Department of Physics, Faculty of Science, Universiti Putra Malaysia, 43400 UPM Serdang, Selangor, Malaysia

Department of Modern Sciences and Technologies, School of Medicine, Mashhad University of Medical Sciences, Mashhad, Iran

Department of Physics, Faculty of Science, Universiti Putra Malaysia, 43400 UPM Serdang, Selangor, Malaysia

We have investigated the spatial self-phase modulation of palm oil containing silver nanoparticles (palm oil/Ag-NPs). The study carried out using continuous wave diode pumped solid state laser with wavelength of $405 \mathrm{~nm}$ and power of $50 \mathrm{~mW}$. The strong spatial selfphase modulation patterns were observed that suggest the palm oil/Ag-NPs have a relatively large nonlinear refractive index. The obtained values of nonlinear refractive index were increased with the increase of volume fractions. The observed experimental patterns were also theoretically modeled which are in good agreement with experimental results.

[DOI: http://dx.doi.org/10.2971/jeos.2012.12020]

Keywords: nonlinear optics, nanoparticles, optical materials, laser ablation

\section{INTRODUCTION}

Metal nanoparticles such as silver and gold have many applications in the areas of physics and chemistry. One of these applications is their ability to increase nonlinear optical properties of the materials. This ability is originated from the presence of intense absorption peak in the visible range due to the surface plasmon resonance of electron [1]-[6]. Palm oil is edible plant oil which derived from the fruits of palm trees. The palm oil is highly saturated fatty acid. On the other hand, the amount of saturated fatty acid and unsaturated fatty acid is the same. Hence, at room temperature palm oil is a mixture of solid and liquid equally. The palm oil contains palmitic $44.3 \%$, stearic $4.6 \%$, and myristic $1 \%$. Nevertheless, the saturated of palm kernel oil, soy bean oil, corn oil and coconut oil is higher than palm oil. Oleic monounsaturated $38.7 \%$ and linoleic polyunsaturated $10.5 \%$ are found in palm oil. The presence of long hydrocarbon chains and polar ester bond make it a good choice for stabilizing nanoparticles. The advantages of palm oil are low cost and high oxidative stability [7].

When an intense laser beam propagates within medium with nonlinear refractive index, a spatial phase shift is induced on laser beam by this medium. As a result of this phase shift, a diffraction pattern will appear on the screen behind the sample. This phenomenon is called spatial self-phase modulation (SSPM) and normally happens for laser with no uniform spatial intensity profile like Gaussian beam [8]-[11]. The obtained diffraction patterns are related to the nonlinear refractive index of samples and can be used to obtain nonlinear refractive index of the samples. In this paper, we present the investigation of SSPM from the palm oil/Ag-NPs.

\section{THEORETICAL SIMULATION}

Consider a Gaussian laser beam with a certain wavelength of $\lambda$ is propagating through a nonlinear medium of length $L$ along the $z$-axis direction and the beam-waist position assumed at the origin of the coordinate system. The complex light field amplitude at the entrance of medium can be expressed as [12]:

$$
E\left(r_{1}, z_{0}\right)=E\left(0, z_{0}\right) \exp \left(-\frac{r_{1}^{2}}{\omega_{p}^{2}}\right) \exp \left(-\frac{i k_{0} n_{0} r_{1}^{2}}{2 R}\right)
$$

where $r_{1}$ is the radial coordinate, $z_{0}$ is the location of the medium entrance plane, $E\left(0, z_{0}\right)$ is the electric field of the 
entrance-plane centre of the medium, $k_{0}$ is the free-space wavenumber, $n_{0}$ is responsible for the refractive index of the air around the medium, $\omega_{p}$ presents the beam radius at the medium entrance plane, $R$ expresses the radius of the wavefront curvature in the corresponding position. Since the thirdorder nonlinear optics occurs within the medium by absorbing the incident light, an additional refractive index distribution $\Delta n\left(z, r_{1}\right)$ gives arise proportional to the launched light intensity. After propagating through the nonlinear medium, the transverse phase shift is induced on the exit Gaussian beam that can be expressed as

$$
\Delta \varphi(r)=k_{0} \int_{z_{0}}^{z_{0}+L} \Delta n\left(z, r_{1}\right) d z
$$

where $\Delta n\left(z, r_{1}\right)=n_{2} I\left(z, r_{1}\right)$ according to Kerr nonlinearity effect. Then, the complex amplitude of the electric field on the exit plane of the nonlinear medium can be written as

$$
\begin{aligned}
& E\left(r_{1}, z_{0}+L\right) \\
= & E\left(0, z_{0}\right) \exp \left(-\frac{\alpha L}{2}\right) \exp \left(-\frac{r_{1}^{2}}{\omega_{p}^{2}}\right) \exp \left(-i \phi\left(r_{1}\right)\right)
\end{aligned}
$$

where $\alpha$ is the linear absorption coefficient and

$$
\begin{aligned}
& \varphi\left(r_{1}\right)=k_{0} \frac{n_{0} r_{1}^{2}}{2 R}+\Delta \varphi\left(r_{1}\right)=k_{0}\left[\frac{n_{0} r_{1}^{2}}{2 R}+\int_{z_{0}}^{z_{0}+L} \Delta n\left(z, r_{1}\right) d z\right] \\
\approx & k_{0} \frac{n_{0} r_{1}^{2}}{2 R}+\Delta \varphi_{0}\left(z_{0}\right) \exp \left(-\frac{2 r_{1}^{2}}{\omega_{P}^{2}}\right)
\end{aligned}
$$

is the total phase shift which arises from the Gaussian phase shift originated by the curvature radius $R$ and the induced transverse additional phase shift after propagating through the medium. In Eq. (4) $\Delta \phi_{0}$, which is proportional to the nonlinear refractive index of used medium, can be evaluated as follow:

$$
\Delta \varphi_{0}=k_{0} \Delta n\left(z_{0}, 0\right) L
$$

Indeed $\Delta \phi_{0}$ denotes the peak of the nonlinear phase-shift induced in the beam which determines the far-field intensity distribution of the Gaussian beam passing through the nonlinear medium. The corresponding far-field intensity distribution of beam after propagation through the sample as a nonlinear medium and free space can be modeled by means of the Fraunhofer approximation of the Fresnel-Kirchhoff diffraction integral:

$$
I=I_{0}\left|\int_{0}^{\infty} J_{0}\left(k_{0} \theta r_{1}\right) \exp \left[-\frac{r_{1}^{2}}{\omega_{P}^{2}}-i \varphi\left(r_{1}\right)\right] r_{1} d r_{1}\right|^{2}
$$

where $I_{0}=4 \pi^{2}\left|E\left(0, z_{0}\right) \exp (-\alpha L / 2) / i \lambda D\right|^{2}, \theta$ is the far field diffraction angle, $J_{0}(x)$ is zero-order Bessel function of the first kind, ris the radial coordinate in the far-field screen of observation plane. In this equation, $D$ is responsible for the distance from the medium exit plane to the screen which is related to the radial coordinate $\rho$ in the screen and the angle $\theta$ by $\rho=D \theta$. Eq. (6) will be studied numerically to assess the law of the farfield diffraction patterns provided by various samples in the following section.

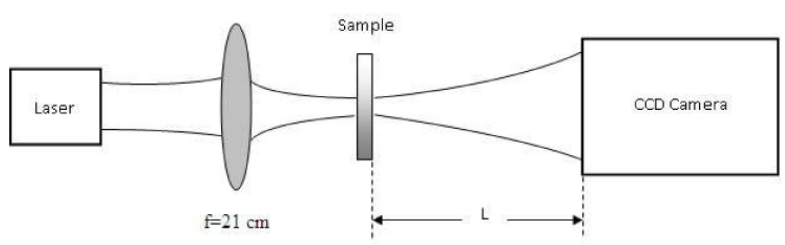

FIG. 1 The experimental set up of SSPM.

\section{EXPERIMENTAL PROCEDURE}

The preparation of Ag-NPs was carried out by laser ablation of pure silver plate in $5 \mathrm{~mm}$ below the surface of $10 \mathrm{~mL}$ of palm oil [13]. The ablation was achieved by blasting the silver plate with a pulsed Q-Switched Nd:YAG laser in constant laser conditions e.g., duration rate $(5 \mathrm{~ns})$, repetition rate (10 Hz), wavelength $(1064 \mathrm{~nm})$, and energy $(360 \mathrm{~mJ} /$ pulse $)$ at different irradiation times $(5,10,15$, and $30 \mathrm{~min})$. A lens with focal length of $250 \mathrm{~mm}$ laser beam was used to focus laser beam on silver target that was located $5 \mathrm{~mm}$ below the surface of oil. The solutions were continuously stirred (magnetically) during the ablation process. The Ag-NPs prepared under the various ablation times were characterized by using UV-vis spectroscopy (Lambda 35, PerkinElmer) and transmission electron microscopy (TEM, Hitachi H-7100). The optical absorption properties of prepared samples were characterized by a UV-vis spectrophotometer and the particle size distributions of nanoparticles from TEM were determined using the UTHSCSA Image Tool Version 3.00 program.

Volume fraction of nanoparticles inside the solution was calculated using the following Eq. (7);

$$
V=\frac{V_{S}}{V_{S}+V_{L}}
$$

where $V_{L}$ is volume of oil and $V_{S}=m / \rho$ is the volume of the particles where $\rho$ is the mass density of the silver and $m$ is the particles mass dispersed in the oil obtained by atomic absorption spectrometer (S Series; Thermo Scientific).

Figure 1 shows the schematic plot of self-diffraction experimental. The $50 \mathrm{~mW}$ diode pumped solid state CW laser with wavelength of $405 \mathrm{~nm}$ was used to observe the SSPM diffraction pattern. A lens with $10 \mathrm{~cm}$ focal length was used to focus laser beam. The sample holder with thickness of $1 \mathrm{~mm}$ was slightly placed after the lens focal length to ensure that the incident beam diverged on the entrance plane of the sample. The far-field diffraction patterns were appeared on a screen behind the sample and recorded by a CCD camera.

The response time of the sample was measured by placing a chopper with variable frequency in front of the lens. The long response time of around $1 \mathrm{~ms}$ was obtained from the beam diffraction pattern which confirms that this phenomenon is due to the thermal effect.

\section{RESULTS AND DISCUSSION}

UV-vis absorption spectra of samples are indicated in Figure 2. The appearance of the sharp peak at around $400 \mathrm{~nm}$ is the evi- 


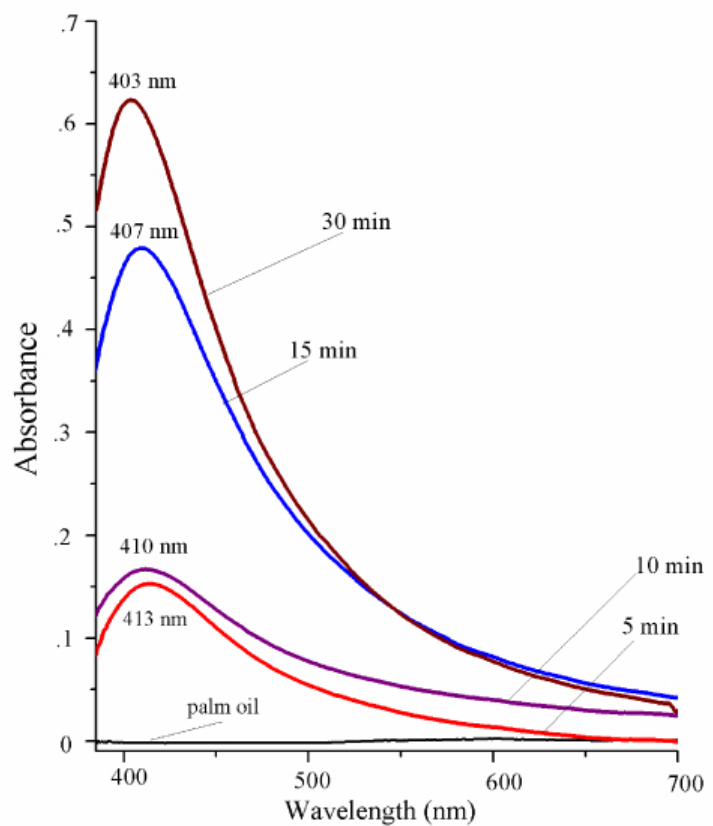

FIG. 2 UV-vis absorption spectra of palm oil/Ag-NPs prepared at different laser ablation times.

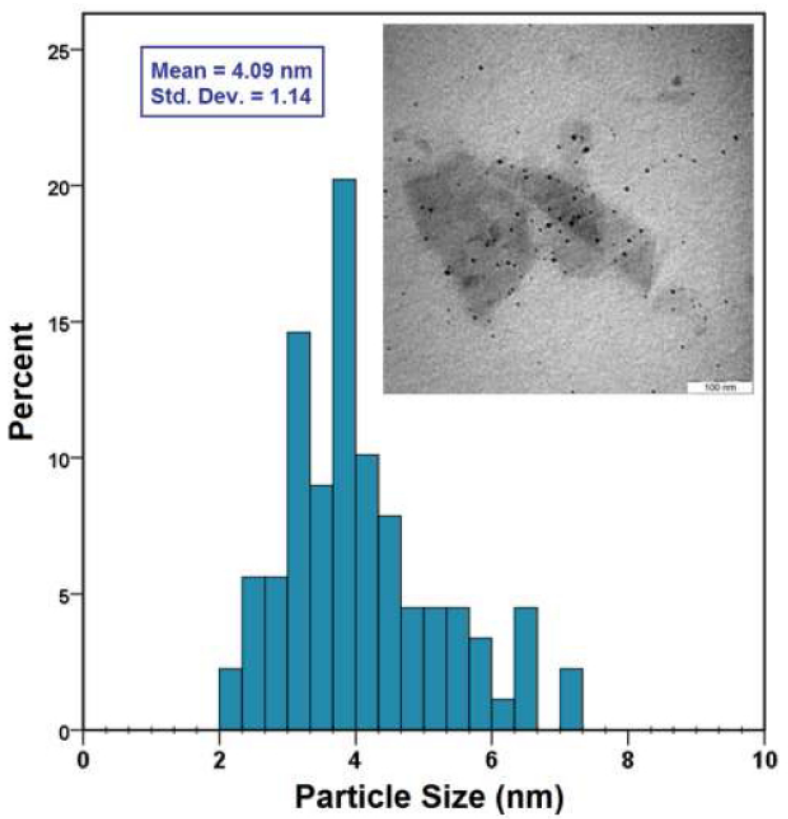

FIG. 3 TEM image and particles size histogram of palm oil/Ag-NPs prepared at $30 \mathrm{~min}$ ablation time.

dent of the Ag-NPs presence in the solutions [14]. As it can be seen from it, by increasing laser ablation time from 5 to $30 \mathrm{~min}$, the intensity of peaks is also increased which is due to increment of nanoparticle formation in the solutions. The position of maximum wavelength $\left(\lambda_{\max }\right)$ of surface plasmon resonance absorption peak at $400 \mathrm{~nm}$ confirms the prepared Ag-NPs are spherical structure [15]. TEM image and corresponding size distributions for the sample prepared at 30 minutes irradiation time is shown in Figure 3. The TEM image also shows that Ag-NPs are well dispersed and spherical shape with mean particles size of about $4 \mathrm{~nm}$.

Figure 4(a)-(d) indicate the practical and numerical far-field diffraction patterns of laser beam after propagating through

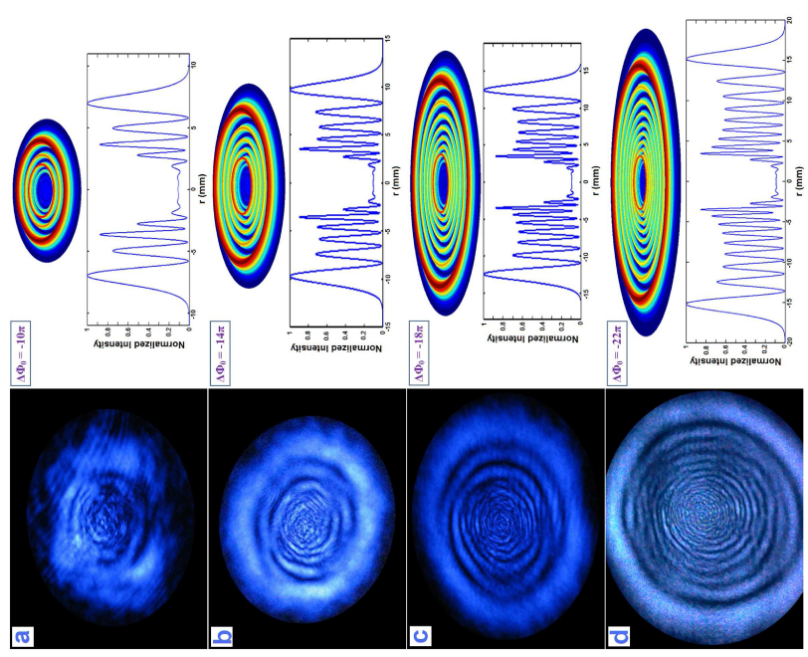

FIG. 4 The experimental and theoretical far-field diffraction patterns at different laser ablation times; (a) 5, (b) 10, (c) 15, and (d) $30 \mathrm{~min}$.

the samples of palm oil/Ag-NPs. To assess the physical implications of the used configuration, this phenomena has been numerically investigated by considering $R=1.33 \mathrm{~m}$ and $D=2.1 \mathrm{~m}$, as the distance between the exit plane of the nonlinear medium and the obtained far-field observational plane, in the following entire computations. The sample holder is located just after the lens focal length $\left(z_{0}\right)$ and as utilized in simulation assumption, the position of beam waist is considered as the origin of the coordinate system. According to the applied standard convention reported in Ref. [12], the applied sample location exposed to the beam light defines that the beam light is divergent at the plane at $z_{0}$ which denotes the positive content of the radius of wave front curvature.

Moreover, the induced phase-shift can also be positive or negative corresponding to this fact that the nonlinear medium acts as a self-focusing or self-defocusing material, respectively. The emerged patterns of the samples as shown in Figure 4(a)(d) are thick diffraction ring pattern with a central dark spot and a relatively large distribution range. This is attributed to this fact that the sign of phase shift is negative and consequently palm oil/Ag-NPs act as a self-defocusing material. It can be observed from the numerical and practical results depicted in Figure 4, the nonlinear phase-shift $\Delta \phi_{0}$ increases linearly with the number of bright diffraction rings $(N)$ around the dark spot increment such that $\left|\Delta \phi_{0}\right|=2 \pi N$ [12]. On the other hand, nonlinear refractive index also increases proportional to the induced phase shift according to equation 5 and thereby the nonlinear refractive index of the applied sample can be calculated as reported in Table 1 . The presence of the large number of rings in the detected diffraction patterns which arises from the induced phase shift indicates a remarkable value of the nonlinear refractive index of the palm oil/Ag-NPs.

According to the obtained results reported in Table 1, the nonlinear refractive index for solvents increases with the increment of nanoparticles volume fraction. We interpret these large nonlinear refractive indexes by the following physics picture. Ag-NPs dispersed in solutions absorb light energy due to SPR of the electron. The absorbed energy will firstly 


\begin{tabular}{|c|c|c|c|}
\hline Laser ablation time $(\mathrm{min})$ & Volume fraction & Number of rings & Nonlinear refractive index, $\boldsymbol{n}_{2}\left(\mathrm{~cm}^{2} / \mathrm{W}\right)$ \\
\hline 5 & $0.7 \times 10^{-8}$ & 5 & $-1.39 \times 10^{-5}$ \\
\hline 10 & $1.0 \times 10^{-8}$ & 7 & $-1.95 \times 10^{-5}$ \\
\hline 15 & $1.7 \times 10^{-8}$ & 9 & $-2.51 \times 10^{-5}$ \\
\hline 30 & $2.8 \times 10^{-8}$ & 11 & $-3.07 \times 10^{-5}$ \\
\hline
\end{tabular}

TABLE 1 The nonlinear refractive index and their corresponding volume fractions of palm oil/Ag-NPs.

transfer to the phonon modes of metal structure of particles and then to the surrounding liquid and then the local temperature will increase in the solution. For most liquids the local refractive index decreases with the increase of temperature therefore temperature gradient results in a change of the local refractive index of solution. In the case of irradiation by $405 \mathrm{~nm}$ CW laser which is near the resonance absorption peak of Ag-NPs, SPR is intense and heat is effectively accumulated. This effect leads to the creation of large thermal-induced negative nonlinear refractive index inside the solutions. On the other hand, when the volume fraction of particles inside the solution is high the absorption of light by SPR is stronger and thereby it causes the increment of the nonlinear refractive index with increase of volume fraction of the particles.

\section{CONCLUSION}

In summary, we have studied SSPM properties of palm oil/Ag-NPs. The samples were prepared by using laser ablation technique under different ablation time. The strong far field diffraction patterns obtained by palm oil/Ag-NPs imply a large value of the induced nonlinear phase-shift. The theoretical simulation also has been studied which shows a good agreement with the experimental explored diffraction patterns and leads a comprehensive survey. The calculated nonlinear refractive index according to the obtained ring numbers of the induced phase-shift indicates a remarkable value which strongly depends on the volume fractions of particles. Therefore, the characterized palm oil/Ag-NPs offer a promising candidate of nonlinear optical material for photonic devices.

\section{References}

[1] V. M. Shalaev, E. Y. Poliakov, and V. A. Markel, "Small particles composites II. nonlinear optical properties," Phys. Rev. B 53, 2437-2449 (1996).

[2] R. A. Ganeev, A. I. Ryasnyanski, A. L. Stepanov, T. Usmanov, C. Marques, R. C. da Silva, and E. Alves, "Investigation of the nonlinear optical characteristics of composite materials based on sapphire with silver, copper, and gold nanoparticles by the reflection Z-scan method," Opt. Spectrosc. 101, 615-622 (2006).

[3] S. Debrus, J. Lafait, M. May, N. Pincon, D. Prot, C. Sella, and J. Venturini, "Z-scan determination of the third-order optical nonlinearity of gold: silica nanocomposites," J. Appl. Phys. 88, 4469-4475 (2000).

[4] X. Qu, J. Wang, C. Yao, and Z. Zhang, "Two-photon imaging of lymphoma cells targeted by gold nanoparticles," Chin. Opt. Lett. 6, 879-881(2008).

[5] C. Wang, Y. Fu, Z. Zhou, Y. Cheng, and Z. Xu, "Femtosecond filamentation and supercontinuum generation in silver-nanoparticle-doped water," Appl. Phys. Lett. 90, 181119-181122 (2007).

[6] E. L. Falcao-Filho, C. B. de Araujo, J. J. Rodrigues, "High-order nonlinearities of aqueous colloids containing silver nanoparticles," J. Opt. Soc. Am. B 24, 2948-2956 (2007).

[7] X. Bo, X. Guomin, Cui. Lingfeng, W. Ruiping, and G. Lijing, "Transesterification of palm oil with methanol to biodiesel over a $\mathrm{kf} / \mathrm{Al}_{2} \mathrm{O}_{3}$ heterogeneous base catalyst," Energ. Fuel. 21, 3109-3112 (2007).

[8] M. A. R. C. Alencar, C. M. Nascimento, S. Chavez-Cerda, M. G. A. da Silva, M. R. Meneghetti, and J. M. Hickmann, “Large spatial self-phase modulation in castor oil enhanced by gold nanoparticles," SPIE 6103, 610306 (2006).

[9] S. D. Durbin, S. M. Arakelian, and Y. R. Shen, "Laser-induced diffraction rings from a nematic-liquid-crystal film," Opt. Lett. 6, 411-413 (1981).

[10] R. Zamiri, A. Zakaria, M. B. Ahmad, A. R. Sadrolhosseini, K. Shameli, M. Darroudi, and M. A. Mahdi, "Investigation of spatial self-phase modulation of silver nanoparticles in clay suspension," Optik 122, 836-838 (2011).

[11] R. Zamiri, B. Z. Azmi, M. Darroudi, A. R. Sadrolhosseini, M. S. Husin, Z. A. Wahab, and M. A. Mahdi, "Preparation of starch stabilized silver nanoparticles with spatial self-phase modulation properties by laser ablation technique," Appl. Phys. A 102, 189-194 (2011).

[12] L. Deng, K. He, T. Zhou, and C. Li, "Formation and evolution of far-field diffraction patterns of divergent and convergent Gaussian beams passing through self-focusing and self-defocusing media," J. Opt. A-Pure Appl. 0p. 7, 409-415 (2005).

[13] R. Zamiri, A. Zakaria, H. A. Ahangar, A. R. Sadrolhosseini, and M. A. Mahdi, "Fabrication of Silver Nanoparticles Dispersed in Palm Oil Using Laser Ablation," Int. J. Mol. Sci. 11, 4764-4770 (2010).

[14] F. Mafune, J. Kohno, Y. Takeda, and T. Kondow, "Formation and size control of silver nanoparticles by laser ablation in aqueous solution," J. Phys. Chem. B 104, 9111-9117 (2000).

[15] J. Xie, J. Y. Lee, D. I. C. Wang, and Y. P. Ting, "Silver nanoplates: from biological to biomimetic synthesis," ACS Nano 1, 429-439 (2007). 